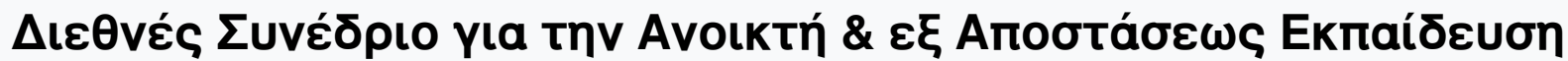

Tóp. 6, Ap. 2B (2011)

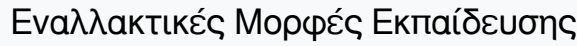

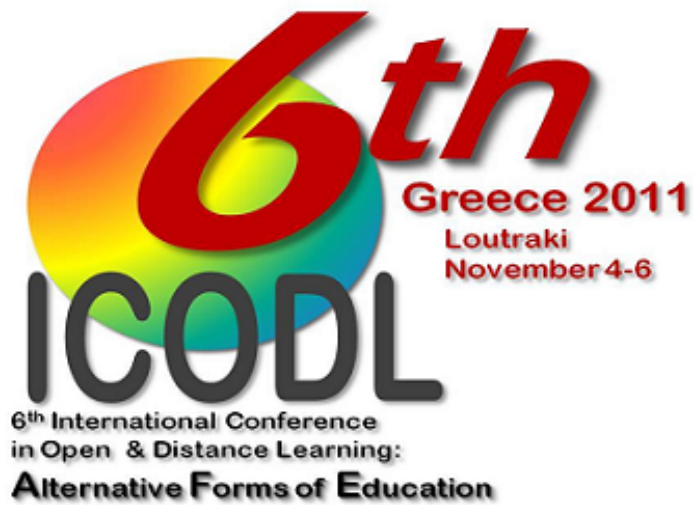

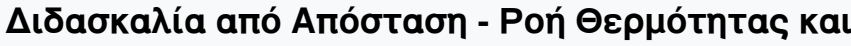

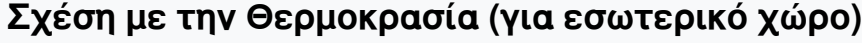

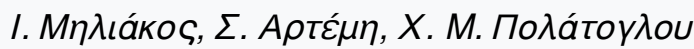

doi: $10.12681 /$ icodl. 655

TOMOE B

PART / MEPOE B 


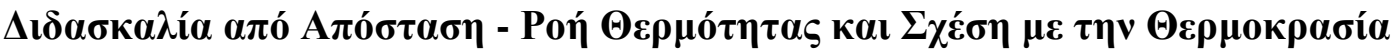

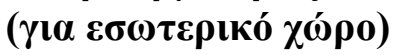

\section{E learning Physics: a didactic simulation about heat flow and temperature difference between the environment and a house}

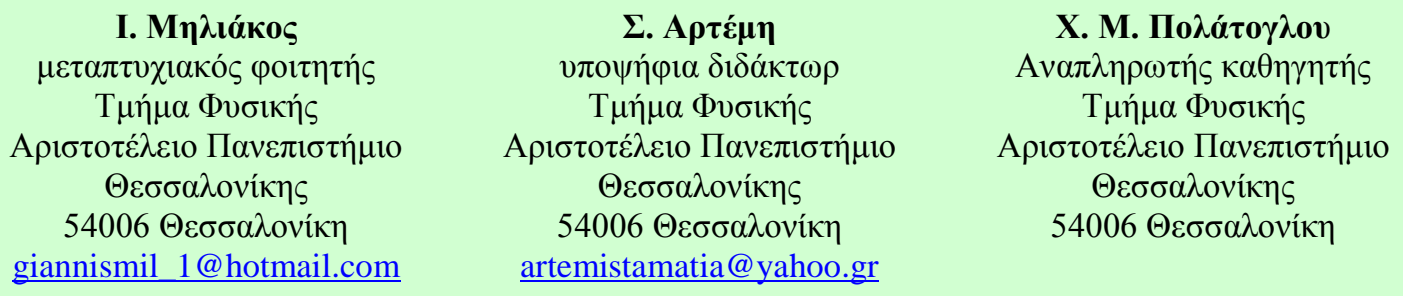

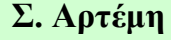

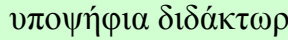 \\ Т $\mu \eta \dot{\mu \alpha}$ Фvбıкท́

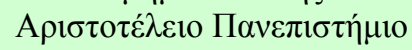

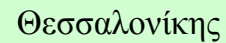 \\ $54006 \Theta \varepsilon \sigma \sigma \alpha \lambda$ ovík \\ artemistamatia@yahoo.gr
}

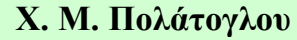

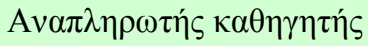 \\ Т $\mu \eta \dot{\mu} \alpha$ Фvбıќ́

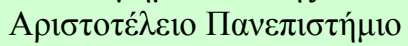 \\ $\Theta \varepsilon \sigma \sigma \alpha \lambda o v i ́ \kappa \eta \varsigma$

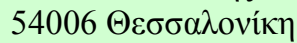

\section{Пврі́ $\eta \psi \eta$}

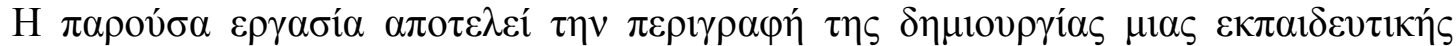

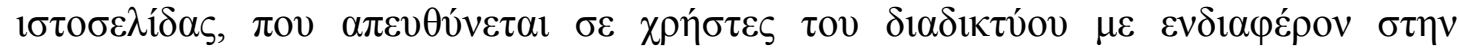

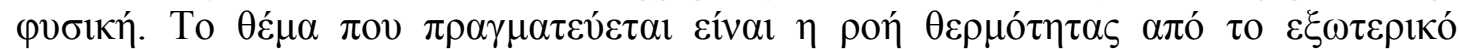

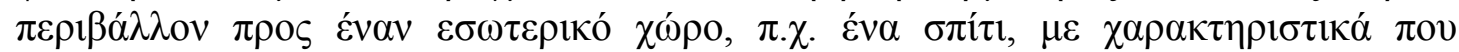

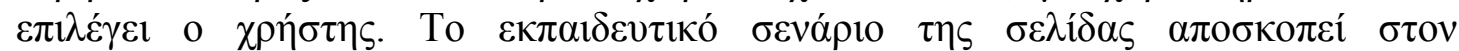

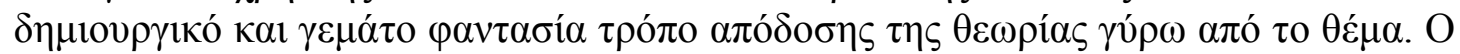

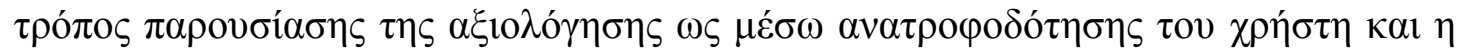

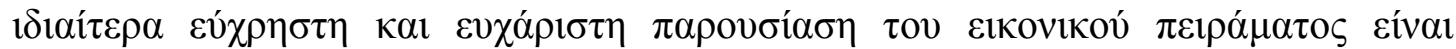

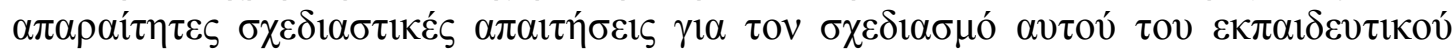

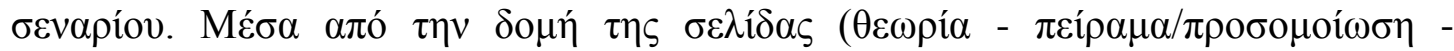

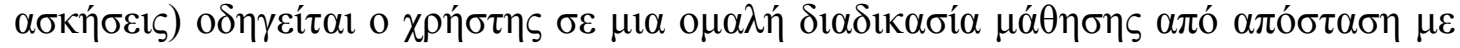

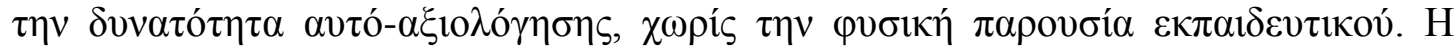

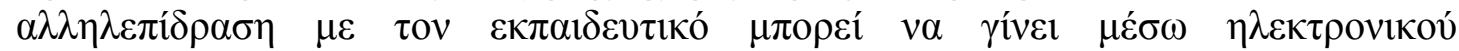
$\tau \alpha \chi v \delta \rho o \mu \varepsilon i ́ o v$.

\begin{abstract}
In this paper we describe the creation of an educational website for internet users with an interest in Physics. The context of this website is the heat flux from the external environment to an interior space, for example a house, which features are chosen by the user. The educational scenario aims at a creative and imaginative rendering of the theory on the subject. The presentation of the evaluation of the user's predictions on the outcome of the experiment, as a mean of the user's feedback, and the easy and pleasant presentation of the virtual experiment design were the requirements for the design of the training scenario. Through the page structure (theory - experiment / simulation - exercises), the user is guided through a smooth process of learning from a distance, with the possibility of self-evaluation, without the physical presence of a teacher. The interaction with the teacher can be done by e mail.
\end{abstract}

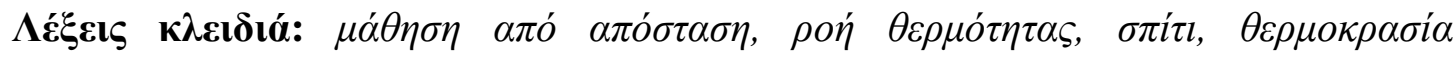

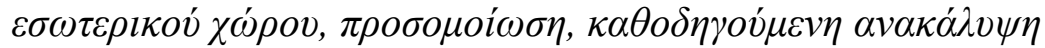




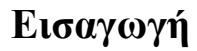

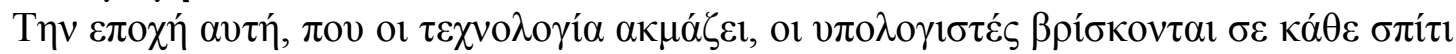

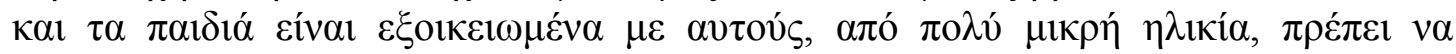

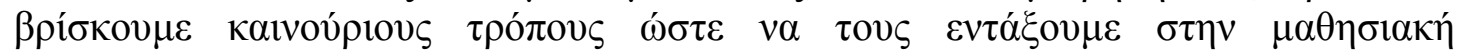

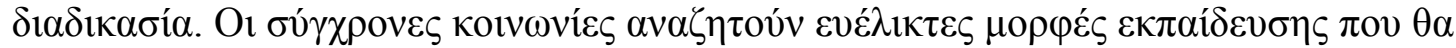

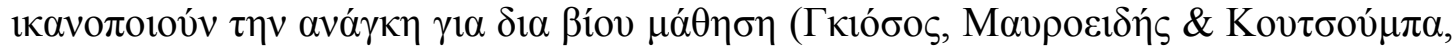

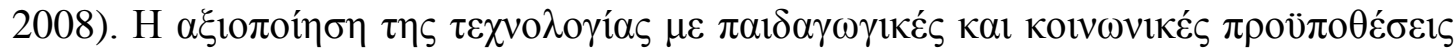

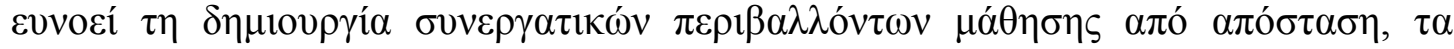

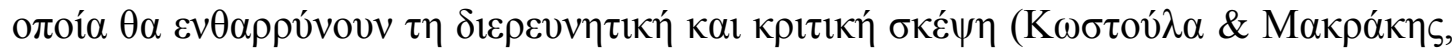

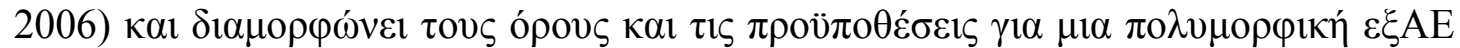

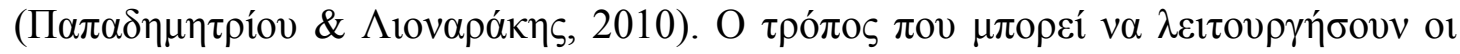

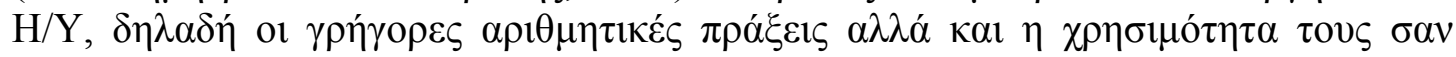

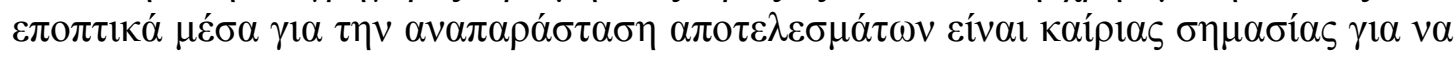

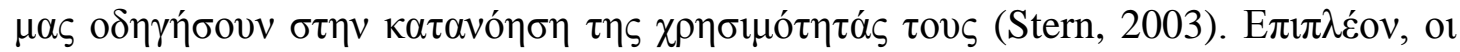

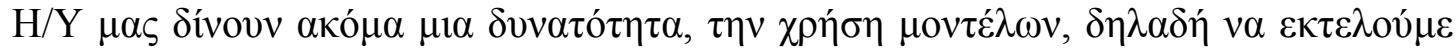

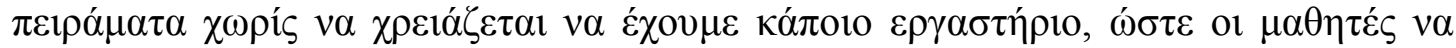

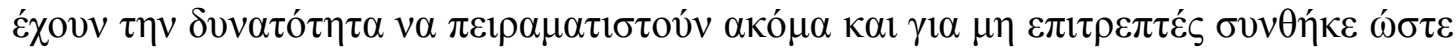

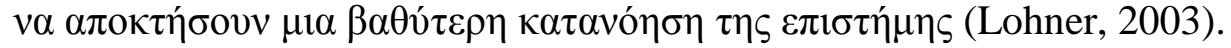

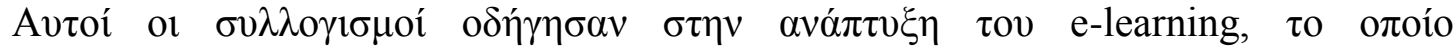

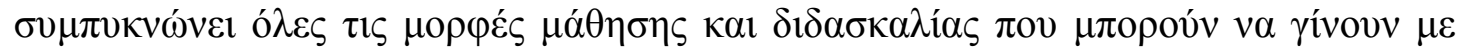

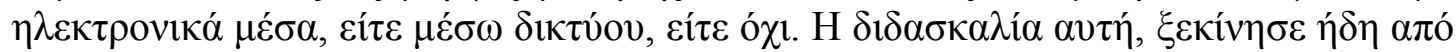

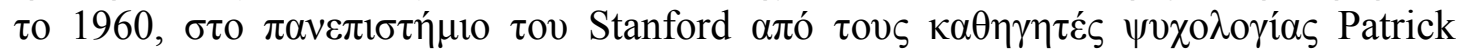

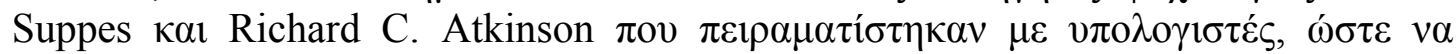

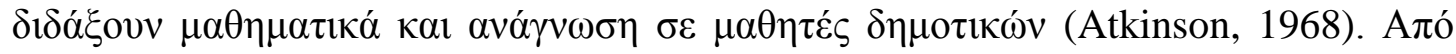

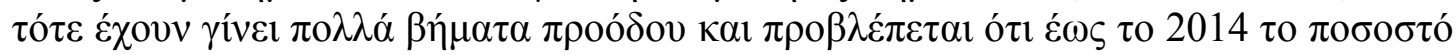

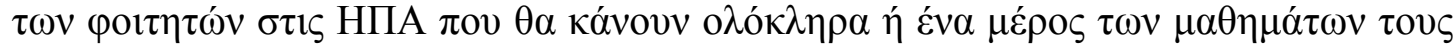

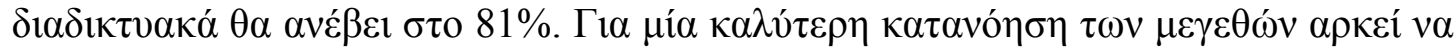

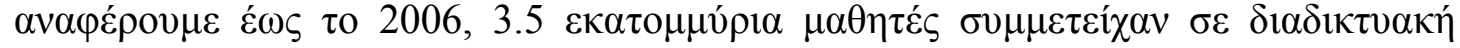

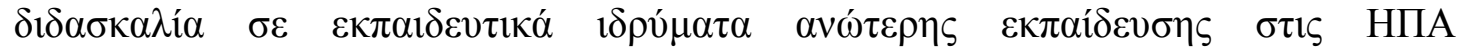

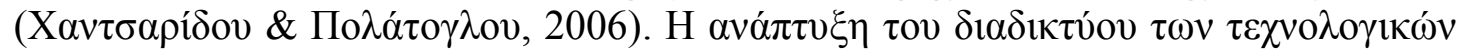

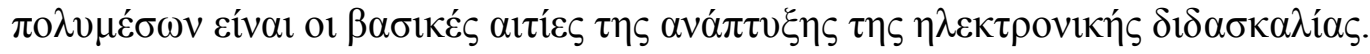

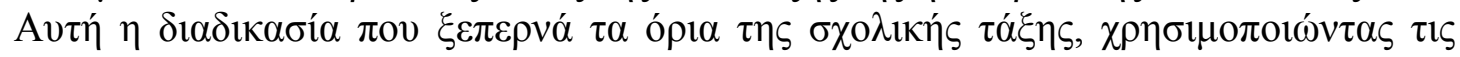

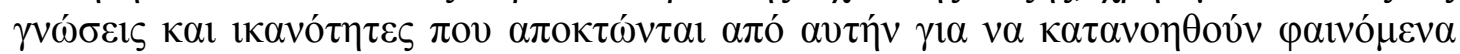

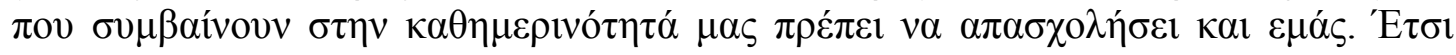

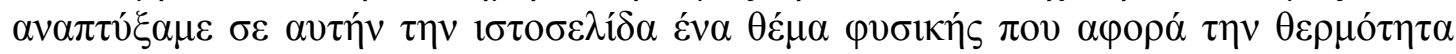

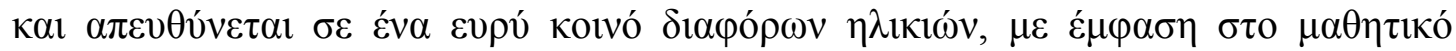

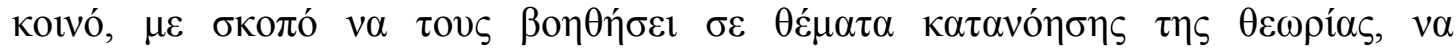

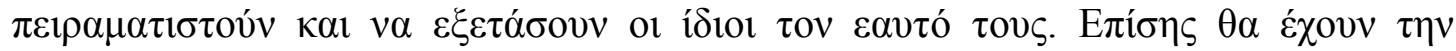

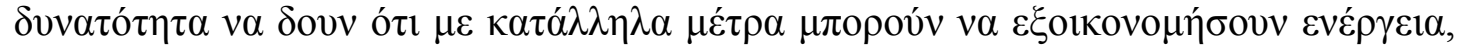

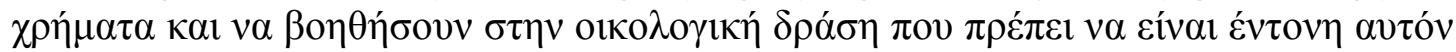

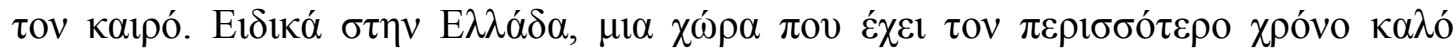

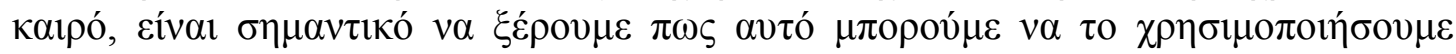

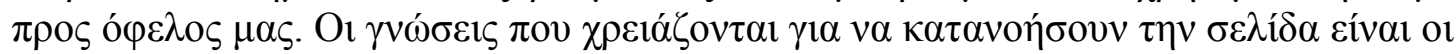

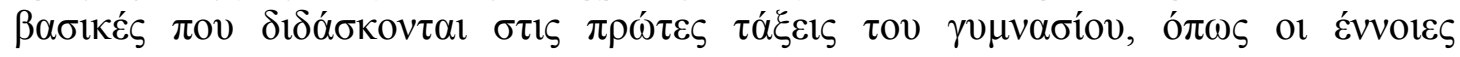
$\varepsilon v \varepsilon ́ \rho \gamma \varepsilon 1 \alpha, \theta \varepsilon \rho \mu o ́ \tau\rceil \tau \alpha, \theta \varepsilon \rho \mu о \kappa \rho \alpha \sigma i ́ \alpha$.

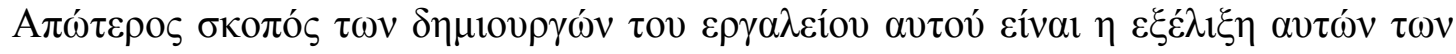

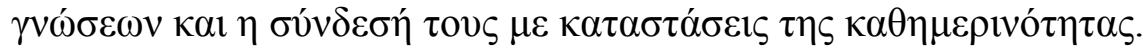




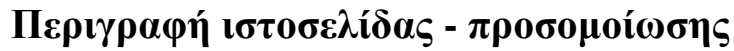

H

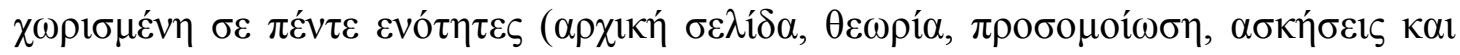
$\varepsilon \pi ı$ เ

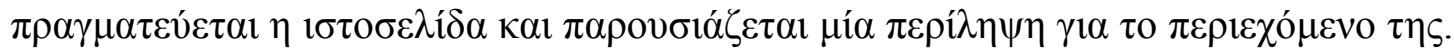

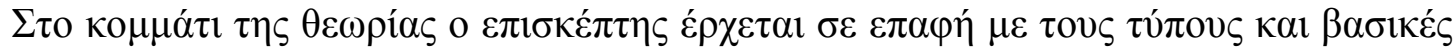

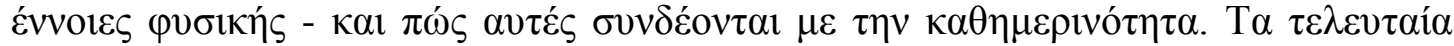

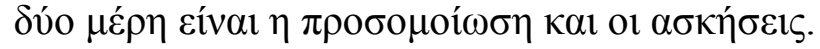

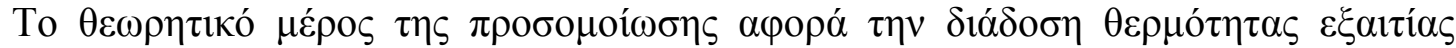

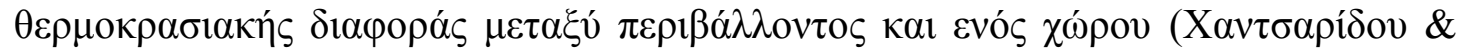

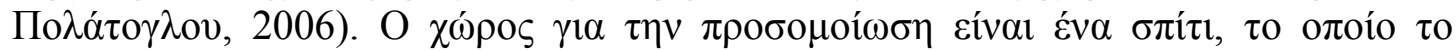

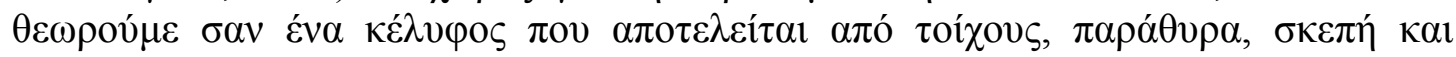

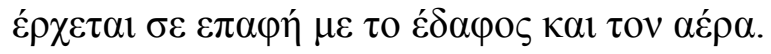

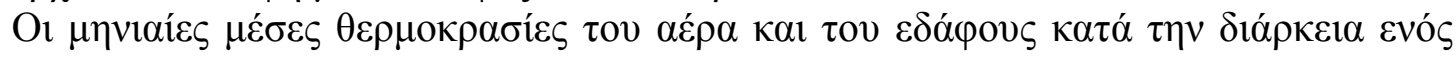

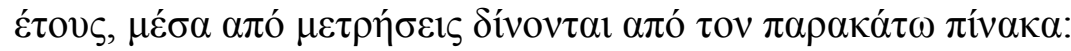

\begin{tabular}{|c|c|c|}
\hline Mи́vas & 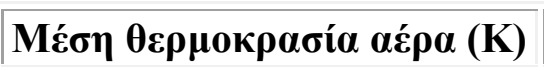 & 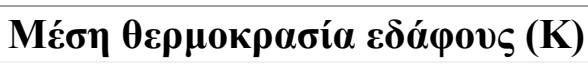 \\
\hline Iavovápios & 282.16 & 281.16 \\
\hline 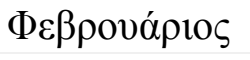 & 282.66 & 283.16 \\
\hline 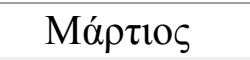 & 286.16 & 286.16 \\
\hline A $\pi \rho i ́ \lambda 10 \varsigma$ & 289.16 & 290.66 \\
\hline Málos & 294.66 & 298.16 \\
\hline Ioúvios & 299.66 & 302.66 \\
\hline 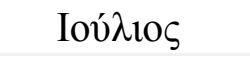 & 301.16 & 305.66 \\
\hline 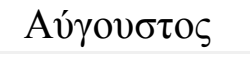 & 300.66 & 305.16 \\
\hline$\Sigma \varepsilon \pi \tau \varepsilon ́ \mu \beta \rho ı \varsigma$ & 296.66 & 299.16 \\
\hline 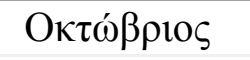 & 292.16 & 292.66 \\
\hline Noé $\mu \beta \rho ı \varsigma$ & 287.16 & 287.16 \\
\hline 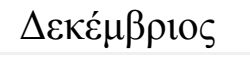 & 284.66 & 283.16 \\
\hline
\end{tabular}

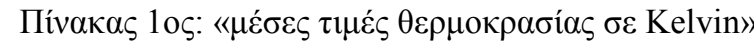
(http://www.physicalgeography.net/fundamentals/71.html)

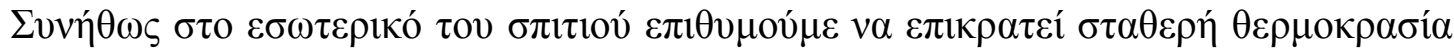

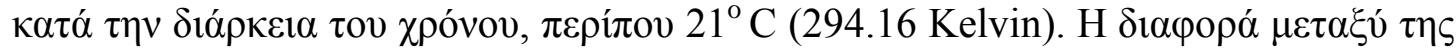

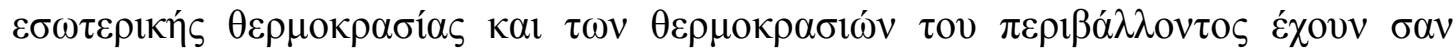

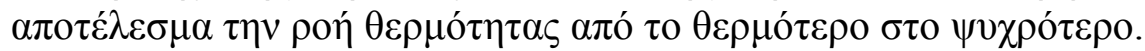

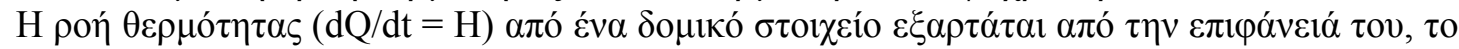

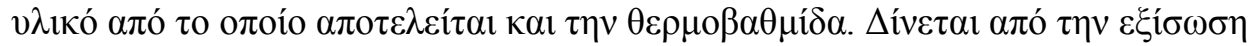

$$
H=k A \frac{\Delta T}{\Delta x}(\mathbf{1})
$$

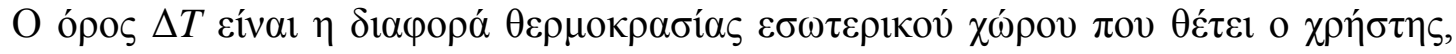

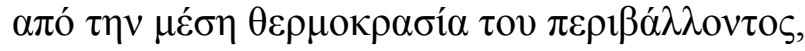




$$
\Delta T=T_{\varepsilon \sigma \omega \tau}-T_{\varepsilon \xi \omega \tau}
$$

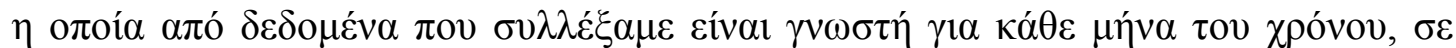

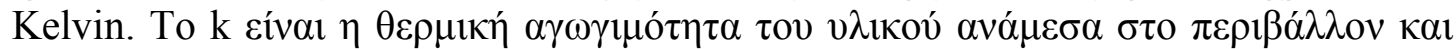

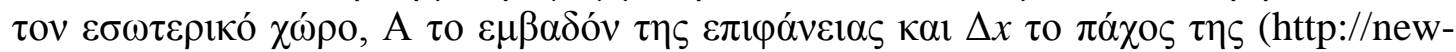
learn.info/learn/packages/clear/thermal/buildings/building_fabric/properties/conductiv

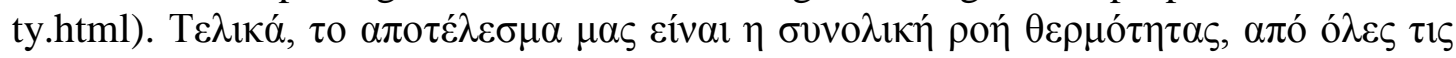

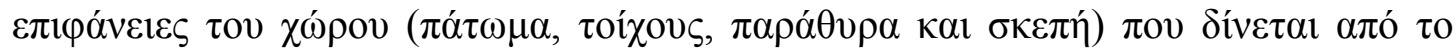

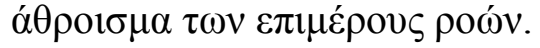

$$
\mathrm{H}_{o \lambda \iota \kappa \dot{\eta}}=\mathrm{H}_{\varepsilon \delta \dot{\alpha} \varphi o v \varsigma}+\mathrm{H}_{\sigma \kappa \varepsilon \pi \dot{\eta} \varsigma}+\mathrm{H}_{\pi \alpha \rho \alpha \theta \dot{v} \rho \omega \nu}+\mathrm{H}_{\tau o i \chi \omega \nu}
$$

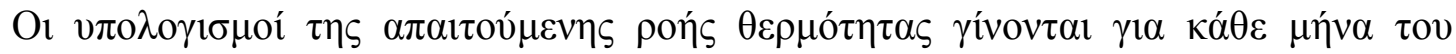

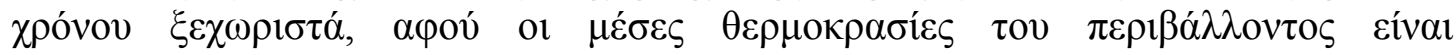

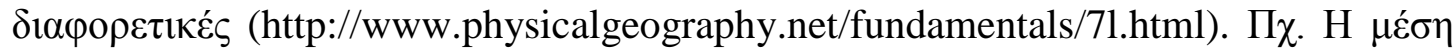

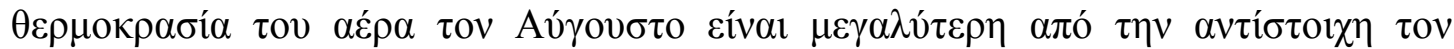

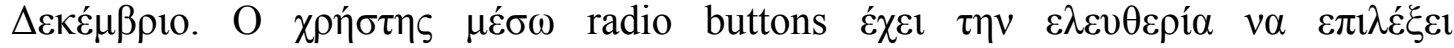

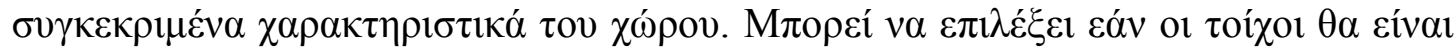

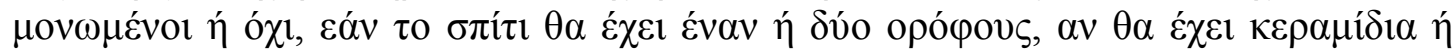

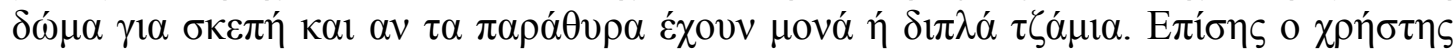

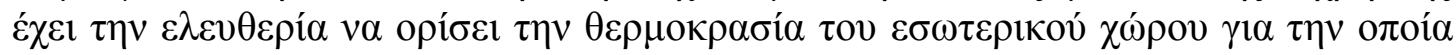

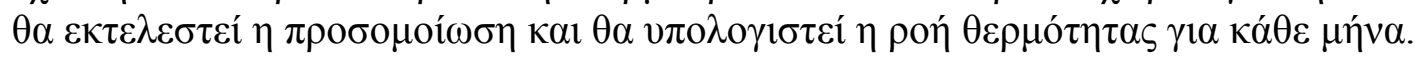

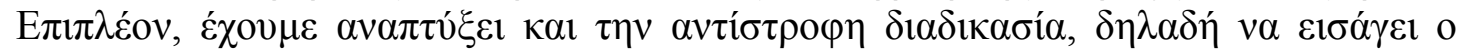

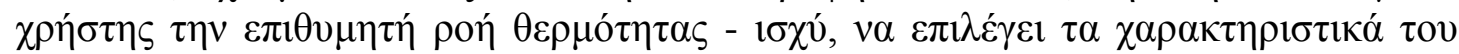

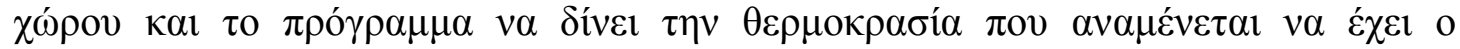

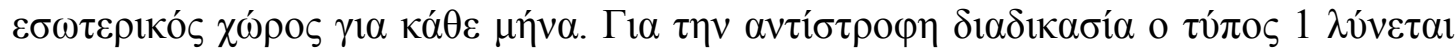
$\omega \varsigma \pi \rho \circ \mathrm{T}_{\varepsilon \sigma \omega \tau}$ :

$$
\mathrm{T}_{\varepsilon \sigma \omega \tau}=\mathrm{T}_{\varepsilon \xi \omega \tau}+\frac{H \Delta x}{k A}
$$

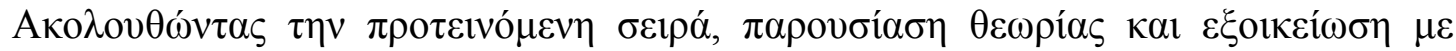

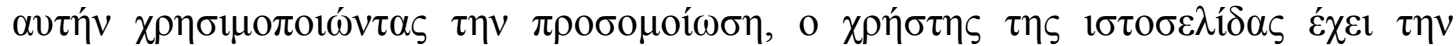

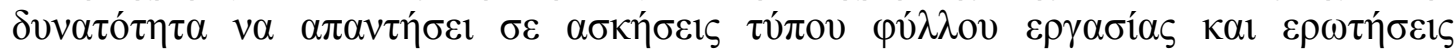

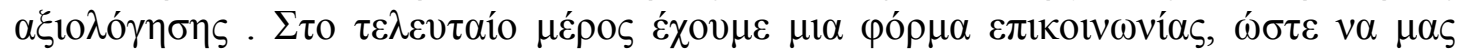

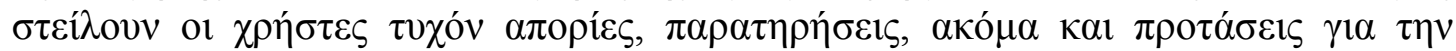

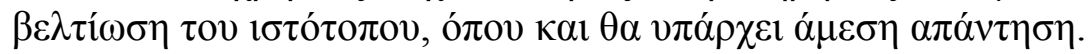

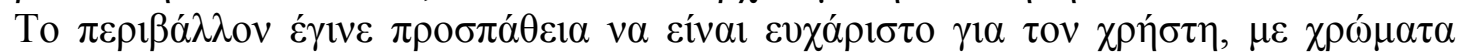

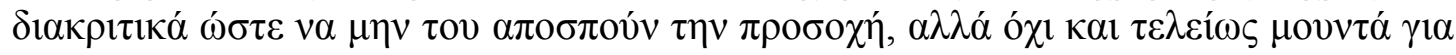

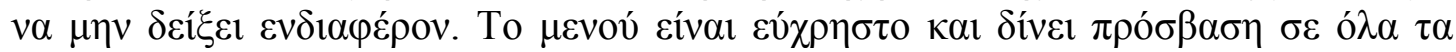

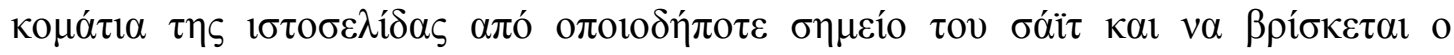
$\varepsilon \pi \imath \sigma \kappa \varepsilon ́ \pi \tau \eta \varsigma$.

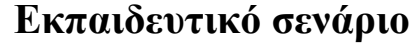

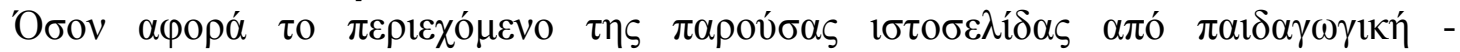

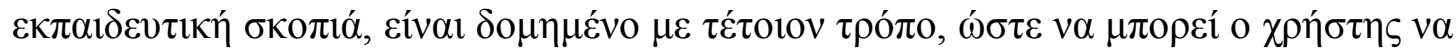

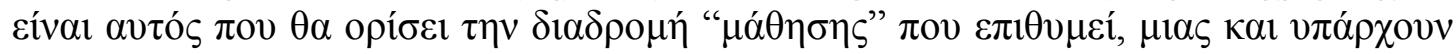




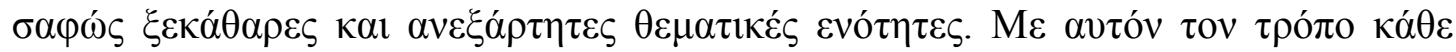

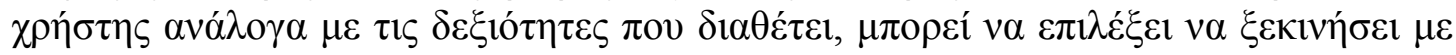

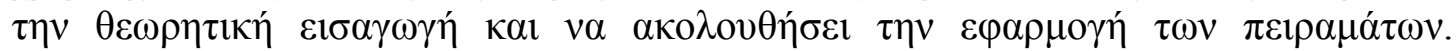

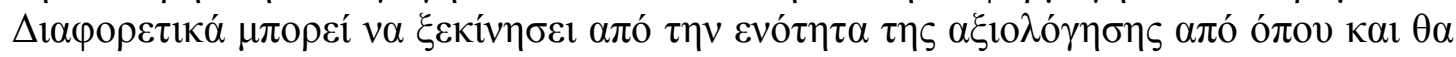

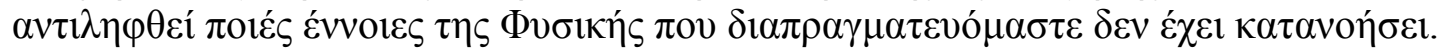

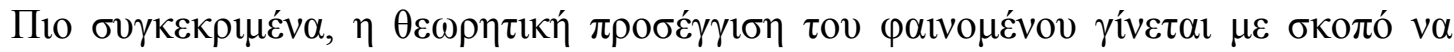

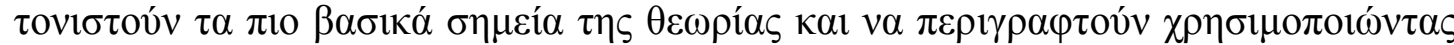

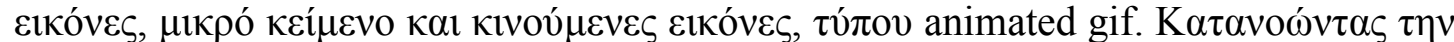

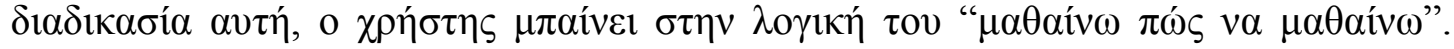

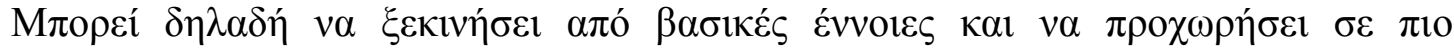

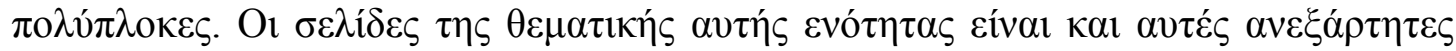

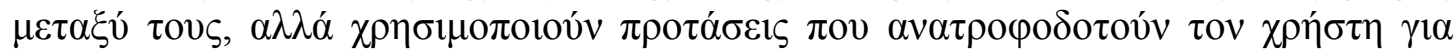

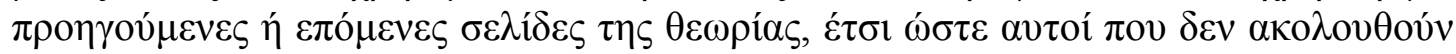

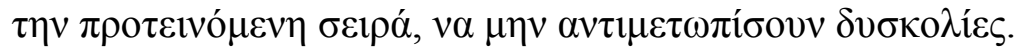

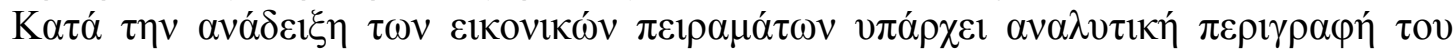

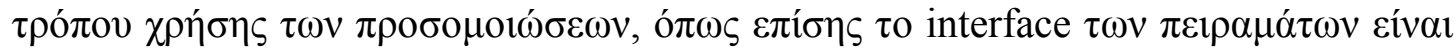

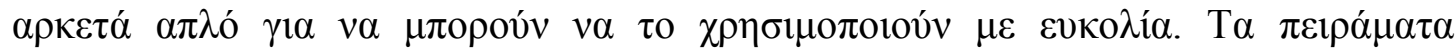

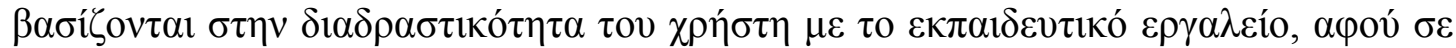

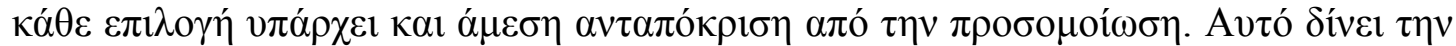

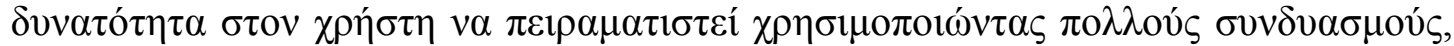

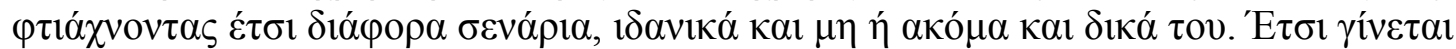

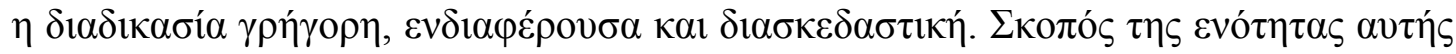

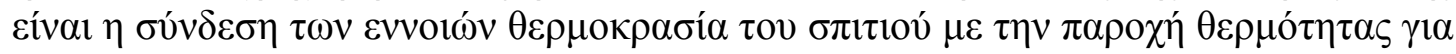

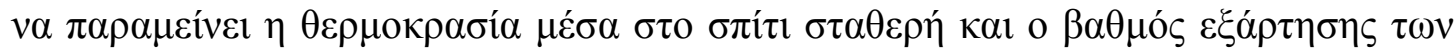

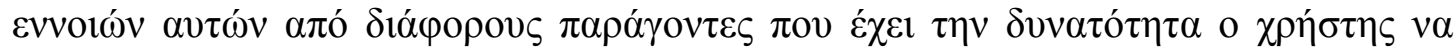

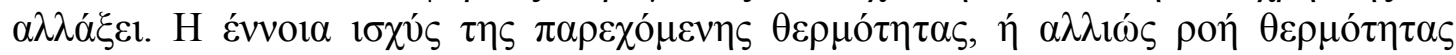

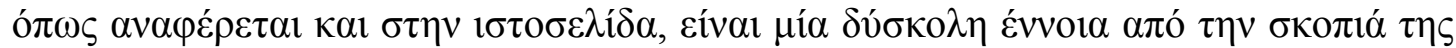

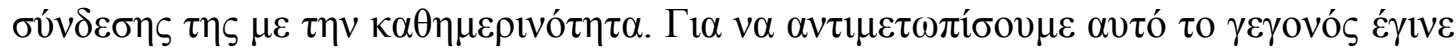

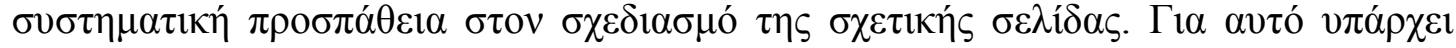

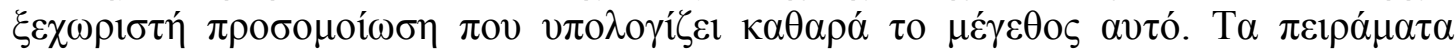

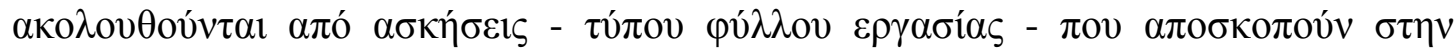

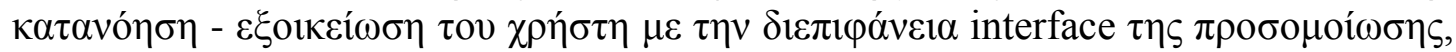

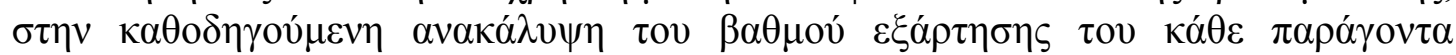

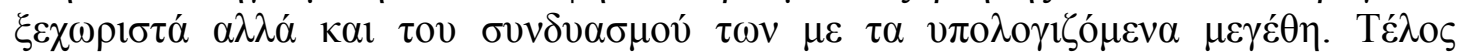

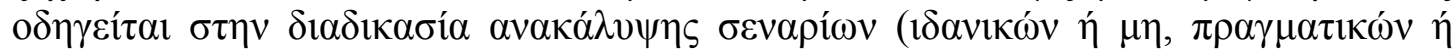

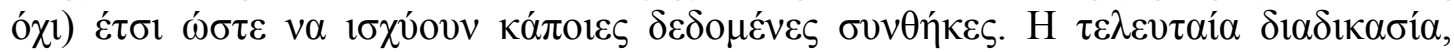

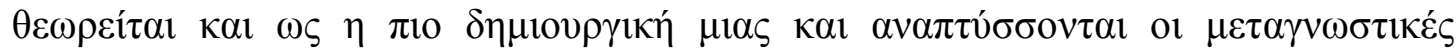

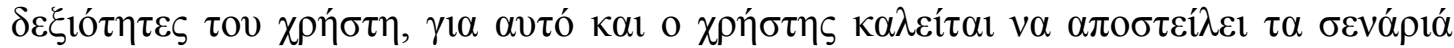

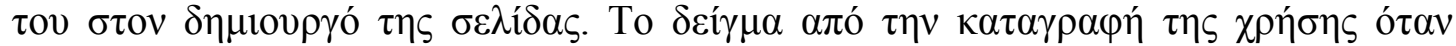

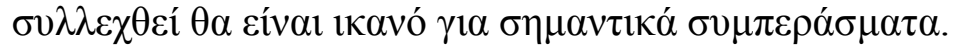

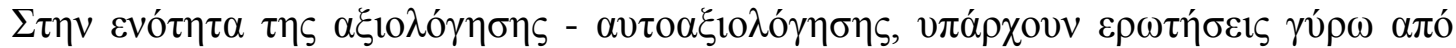

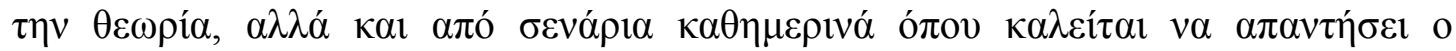

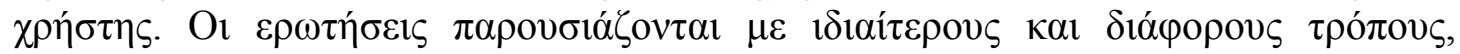

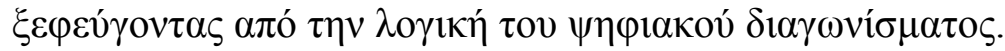

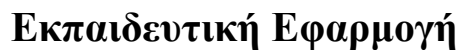

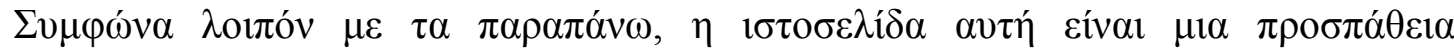

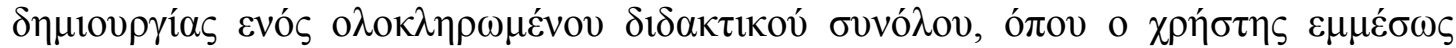

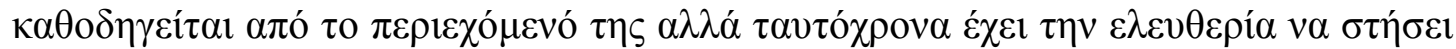

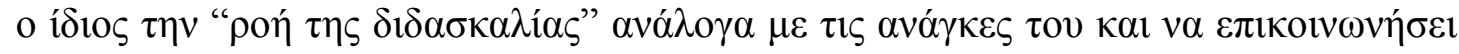




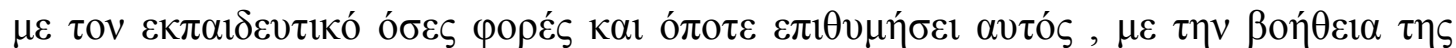

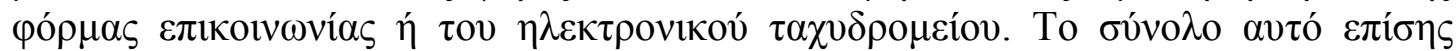

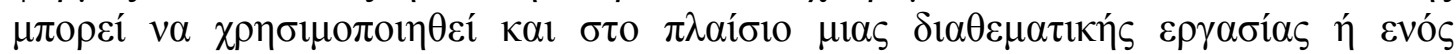

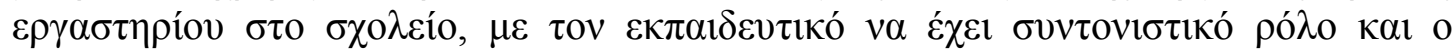

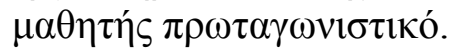

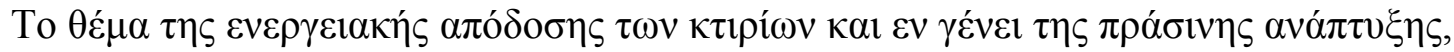

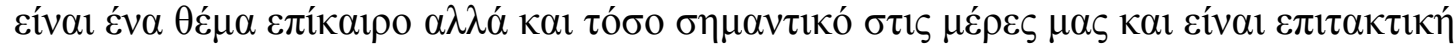

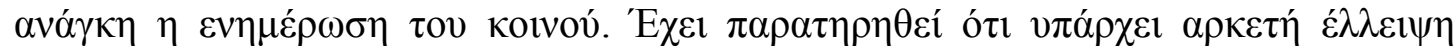

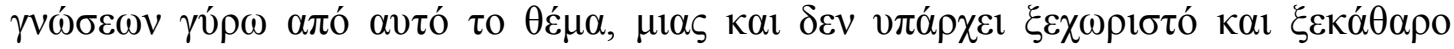

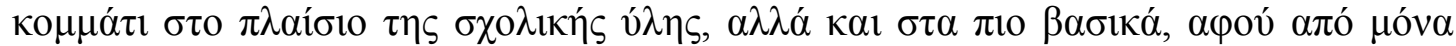

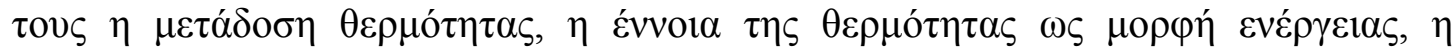

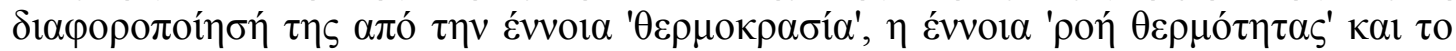

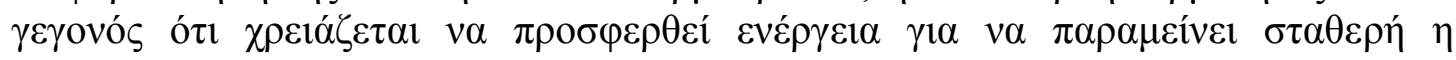

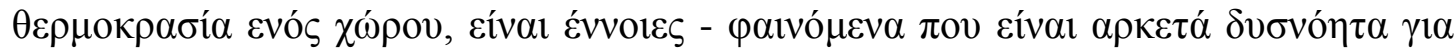

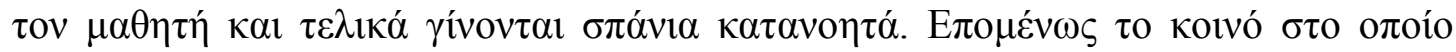

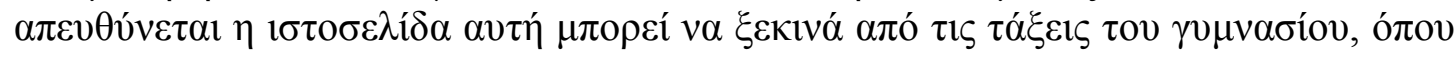

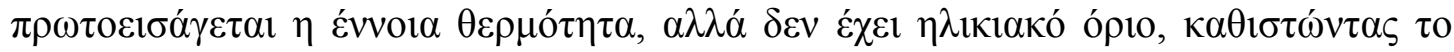

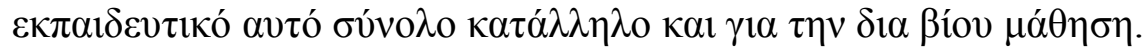

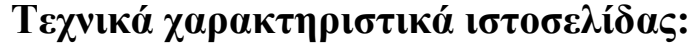

H

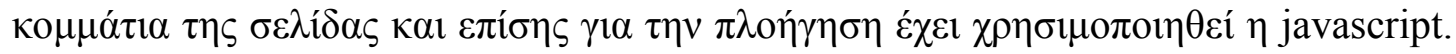
H

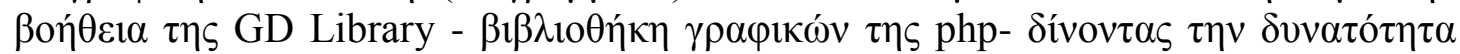

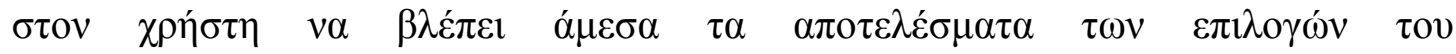

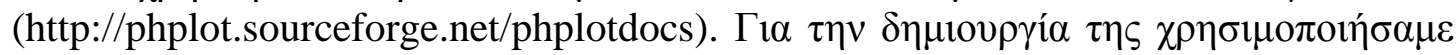
$\tau \alpha \pi \rho \gamma_{\rho \alpha \alpha \mu} \mu \alpha \tau \alpha$ NetBeans $\kappa \alpha$ t $\tau$ o Microsoft Expression Web. To NetBeans

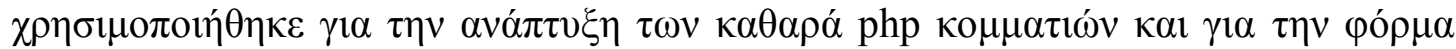

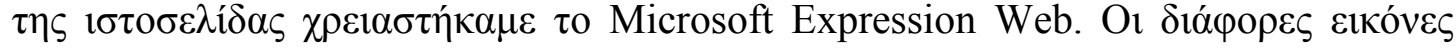

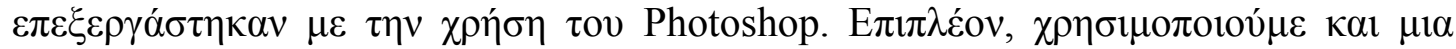

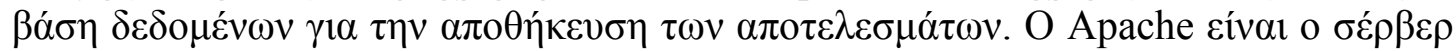

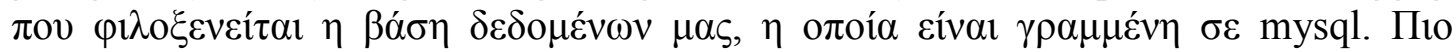

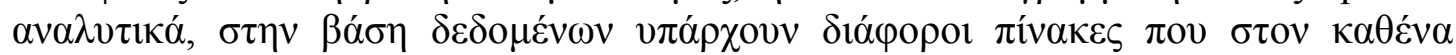

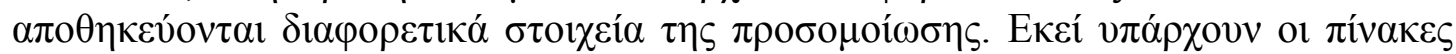

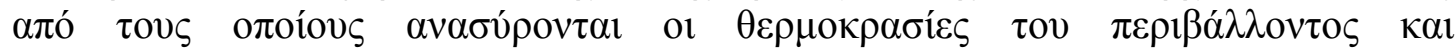

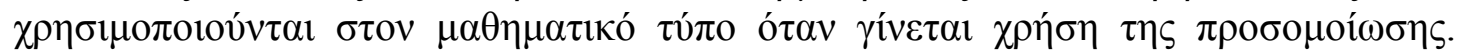

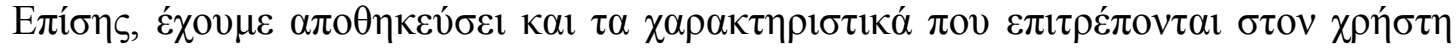

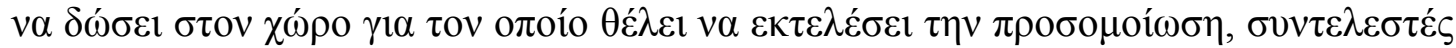

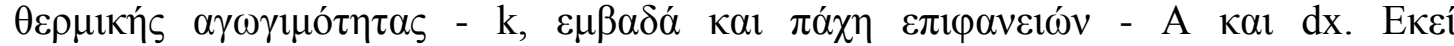

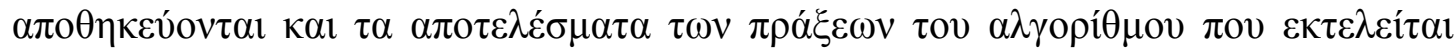

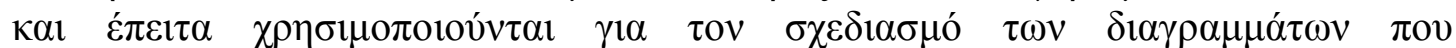

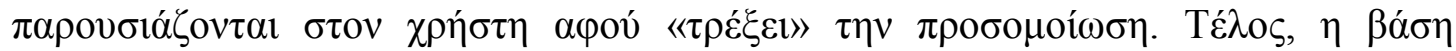

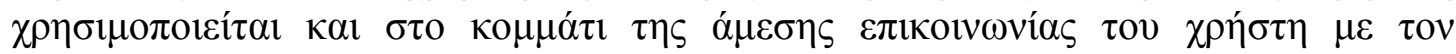

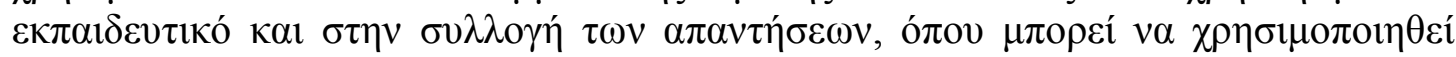

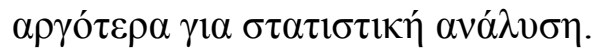




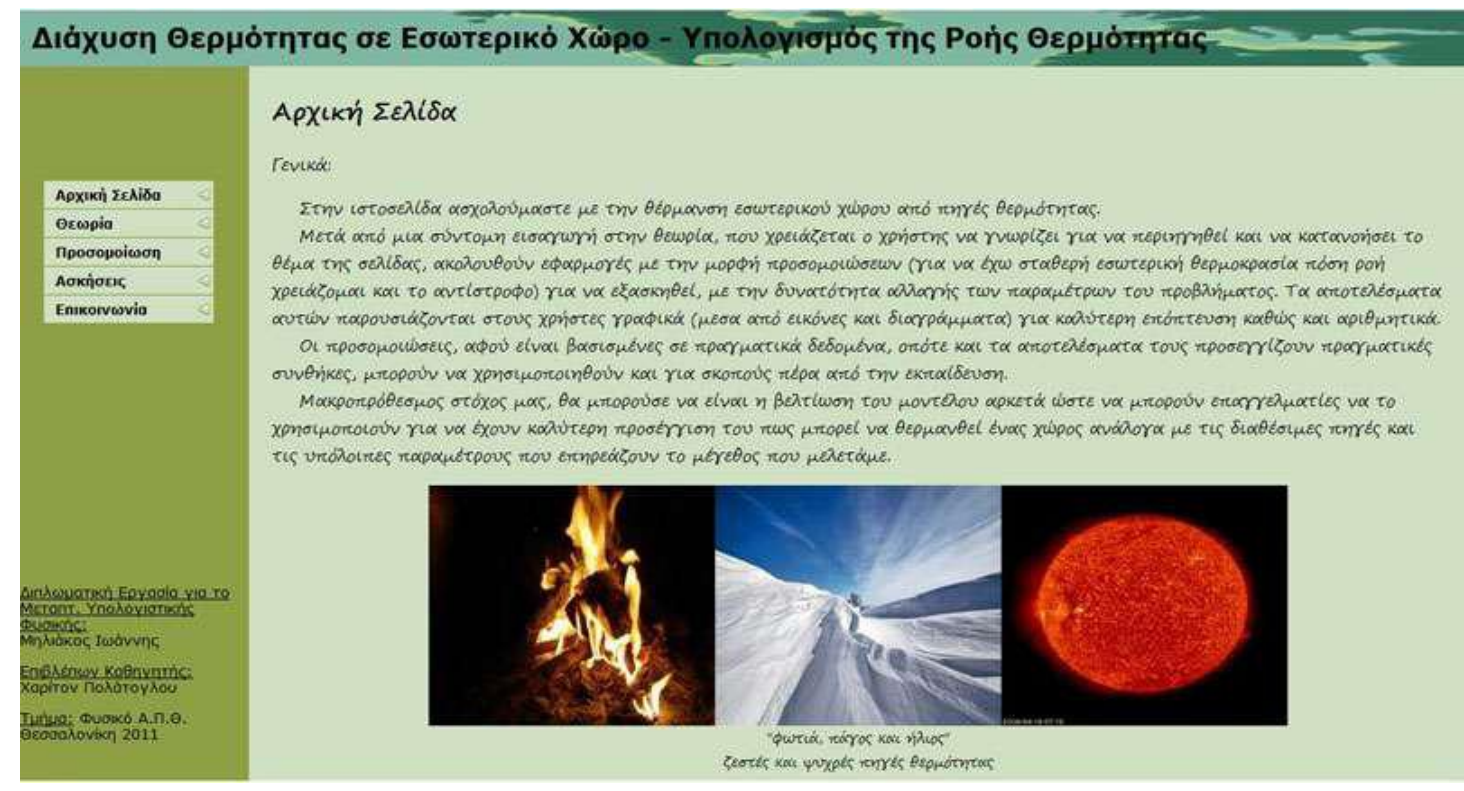

\section{$\Sigma v \mu \pi \varepsilon \rho \alpha ́ \sigma \mu \alpha \tau \alpha$}

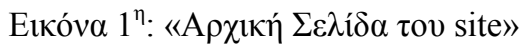

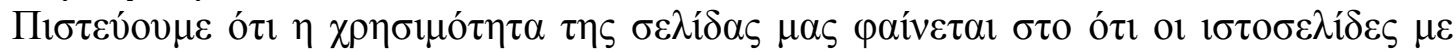

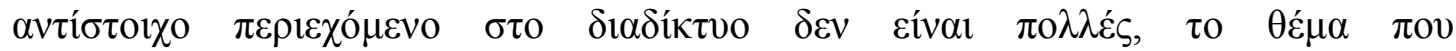

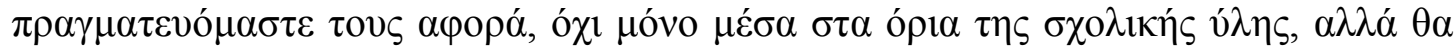

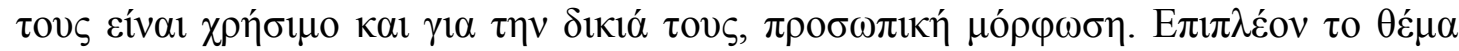

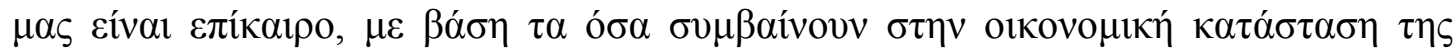

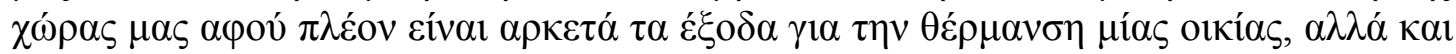

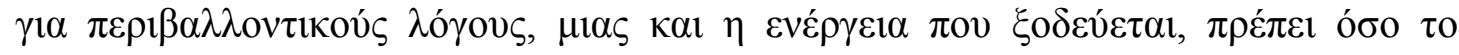

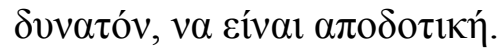

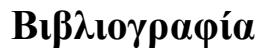

Allen, I. E. and Seaman, J. (2008). Staying the Course: Online Education in the United States. Needham MA, Sloan Consortium

Atkinson, Richard C., (Apr 1968). Computerized instruction and the learning process, American Psychologist, Vol 23(4), pp. 225-239

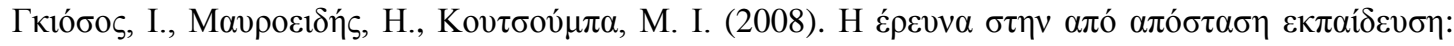

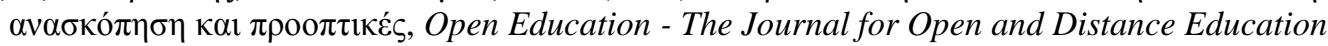
and Educational Technology, Volume 4, Number 1, 2008 / Section one. (C) Open Education ISSN: 1791-9312

Lohner, S, (2003). Instructional Science, 31, pp. 395-418

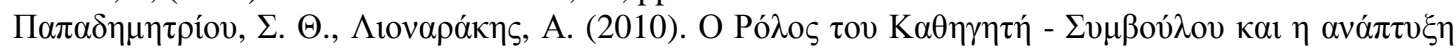

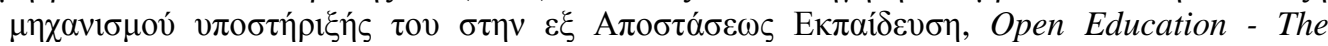
Journal for Open and Distance Education and Educational Technology, Volume 6, Number 1 \& 2, 2010 Section one. (C) Open Education ISSN: 1791-9312

Stern, E, (2003), Learning and Instruction 13, pp. 191-203

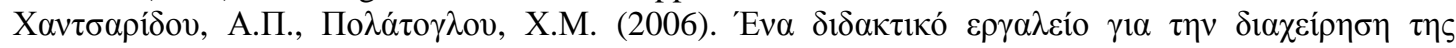
http://new-

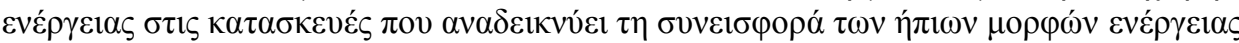

learn.info/learn/packages/clear/thermal/buildings/building_fabric/properties/conductivty.html, Retrieved on April, 2011

http://phplot.sourceforge.net/phplotdocs/, Retrieved on April, 2011

http://www.physicalgeography.net/fundamentals/7l.html, Retrieved on April, 2011 